\title{
The Moon as a photometric calibration standard for microwave sensors
}

\author{
Martin Burgdorf, Stefan A. Buehler, Theresa Lang, Simon Michel, and Imke Hans \\ Meteorologisches Institut, Universität Hamburg, Bundesstraße 55, 20146 Hamburg, Germany \\ Correspondence to: Martin Burgdorf (martin.burgdorf@uni-hamburg.de)
}

Received: 1 February 2016 - Published in Atmos. Meas. Tech. Discuss.: 29 February 2016

Revised: 3 June 2016 - Accepted: 17 June 2016 - Published: 1 August 2016

\begin{abstract}
Instruments on satellites for Earth observation on polar orbits usually employ a two-point calibration technique, in which deep space and an onboard calibration target provide two reference flux levels. As the direction of the deep-space view is in general close to the celestial equator, the Moon sometimes moves through the field of view and introduces an unwelcome additional signal. One can take advantage of this intrusion, however, by using the Moon as a third flux standard, and this has actually been done for checking the lifetime stability of sensors operating at visible wavelengths. As the disk-integrated thermal emission of the Moon is less well known than its reflected sunlight, this concept can in the microwave range only be used for stability checks and intercalibration. An estimate of the frequency of appearances of the Moon in the deep-space view, a description of the limiting factors of the measurement accuracy and models of the Moon's brightness, and a discussion of the benefits from complementing the naturally occurring appearances of the Moon with dedicated spacecraft maneuvers show that it would be possible to detect photometric lifetime drifts of a few percent with just two measurements. The pointing accuracy is the most crucial factor for the value of this method. Planning such observations in advance would be particularly beneficial, because it allows observing the Moon at welldefined phase angles and putting it at the center of the field of view. A constant phase angle eliminates the need for a model of the Moon's brightness when checking the stability of an instrument. With increasing spatial resolution of future microwave sensors another question arises, viz. to what extent foreground emission from objects other than the Moon will contaminate the flux entering the deep-space view, which is supposed to originate exclusively in the cosmic microwave background. We conclude that even the brightest discreet
\end{abstract}

sources have flux densities below the detection limit of microwave sensors in a single scan.

\section{Introduction}

Understanding and predicting climate variation requires accurate measurements of small changes over long periods of time. The global surface temperature, for example, has increased by only $0.113^{\circ} \mathrm{C}$ decade $^{-1}$ over the period 1950 1999 (Karl et al., 2015). Characterizing such trends from space with sufficient accuracy is difficult, because the typical operational lifetime of a satellite rarely exceeds 10 years, and the performance of an instrument might change in unexpected ways in orbit.

Previous studies to intercalibrate microwave sensors on different satellites have concentrated on averaging Earth view data in certain ways or on finding collocations between measurements (e.g., John et al., 2012, 2013). Earth scenes, however, are not absolute calibration targets, since they will change over time, especially in a changing climate. Hence there is a need for an invariable radiometric standard as a reference for checking the stability of the absolute photometric calibration. The object best suited for this purpose is the Moon, which appears occasionally in the deep-space view (DSV) of instruments on satellites for Earth observation on polar orbits. As its spectral hemispherical emissivity is constant for all practical purposes (Kieffer, 1997), only the orbital effects - i.e., the Sun-satellite-Moon angle; the distances between Earth, Moon, and Sun; and libration - can make a difference to the flux arriving in the DSV. After characterizing these variations by taking lunar images for several years with an automated observatory on the ground - 
the ROLO (RObotic Lunar Observatory) telescope (Kieffer and Stone, 2005) - the Moon was established as the radiance standard for wavelengths between 0.35 and $0.95 \mu \mathrm{m}$ (Kieffer and Wildey, 1996). It could be shown that nine lunar comparisons spread over 1 year are sufficient to detect changes, for example of the VIIRS (Visible Infrared Imaging Radiometer Suite) reflective solar bands, at the $0.1 \%$ level (Patt et al., 2005).

The ROLO project cannot easily be extended to longer wavelengths - thermal infrared or microwave - because the brightness of the stars needed for monitoring the calibration of the camera observing the Moon decreases proportional to the square of the wavelength, and the atmosphere is only transparent in certain spectral windows. It is possible, however, to correct for the orbital variations of the Moon's brightness considerably by fitting second-order polynomials of certain orbital variables to the radiances measured with the sensor itself. This method was used to obtain a model for subtracting the lunar contamination in the calibration of AMSU-A (Advanced Microwave Sounding Unit-A; Mo and Kigawa, 2007). Unlike instruments that operate in the visible and infrared spectral range, microwave sensors cannot spatially resolve the Moon. Hence Mo and Kigawa (2007) characterized its microwave brightness temperature with a single value. Maps of the brightness variations over its disk at certain millimeter wavelengths were made, however, with ground-based radio telescopes (Coates, 1961).

Adjusting for orbital effects purely on the basis of the observations with the satellite itself requires a large dataset. Mo and Kigawa (2007) had to put up with unplanned lunar intrusions in the DSV of AMSU-A, which crossed the DSV at quite different distances from its center. It therefore seems desirable to establish a fixed pattern of spacecraft roll maneuvers for future missions like ICI (Ice Cloud Imaging) or MWI (MicroWave Imaging) for MetOp Second Generation (MetOp-SG) in order to measure their lifetime stability by means of observations of the Moon. In the following we make the case for this calibration strategy.

\section{Frequency of the Moon appearing in the deep-space view}

The microwave sensors in space are calibrated in flight by measuring the signal from both the cosmological microwave background (CMB) at $2.72548 \pm 0.00057 \mathrm{~K}$ (without foreground emission; Fixsen, 2009) and an onboard calibration target at some $280 \mathrm{~K}$. The DSV points at a fixed angle $\alpha$ from nadir in the anti-Sun direction and perpendicular to the flight direction (see Fig. 1). $\alpha$ can take values between $65^{\circ}$ and $81^{\circ}$ for AMSU-B, but only a fraction of this range is actually used in operations (Charlton et al., 1993). The corresponding range for MHS (Microwave Humidity Sounder; Goodrum et al., 2014) is $71^{\circ} \leq \alpha \leq 76^{\circ}$. In the course of one orbit of the spacecraft, the deep-space viewing direction describes a circle with an opening angle of $2 \times\left(90^{\circ}-\alpha\right)$ in the sky; so if the DSV were perpendicular to nadir, it would always point in the same direction during one orbit (apart from the movement of the Sun-synchronous orbit itself). The center of this circle is $\approx 9^{\circ}$, i.e., the value of the north-south orbital inclination of the satellite, away from the celestial equator. This position, in combination with the Equator-crossing time (local time) of the spacecraft and the angle $\alpha$, determines the range of phase angles under which the Moon can appear in the DSV.

As the orbit of the Moon around the Earth is tilted by $5.15^{\circ}$ against the ecliptic plane, which in turn is tilted by $23.45^{\circ}$ against the celestial equator, the Moon can be at a given time anywhere between the extreme positions $28.6^{\circ}$ north and south of the Equator. Hence there are periods when the Moon's orbit does not cross the circle described in the sky by the DSV direction and the Moon cannot be seen by the microwave sensor. The larger the angle $\alpha$, the smaller is the opening angle of the DSV circle, and the less often intrusions of the Moon happen on average. The comparison of the frequency of Moon intrusions for different microwave sensors in Table 1 shows that MHS, with its relatively small $\alpha$, typically has the Moon within its DSV ring eight times a year, whereas instruments with higher $\alpha$ are less often affected. Table 2 lists the distance of the Moon from the celestial equator at the times when it approaches the DSV circle during its orbit around the Earth. It can be seen that the Moon can only appear in the DSV if its declination is in the range -4 to $22^{\circ}$ for AMSU-B and -9 to $27^{\circ}$ for MHS, which corresponds to the range covered by the DSV circle $\left(90^{\circ}-\alpha\right.$ around its center at a declination of $+9^{\circ}$ ).

Sometimes there are two groups of contaminated orbits per month, which are separated by 1 or 2 days, corresponding to the Moon's entry and exit in the DSV ring. A lunar intrusion can contaminate as little as $1 \%$ of the scans in an orbit of MHS. Up to a third of all scans in one orbit, however, can be affected by the Moon with AMSU-A. The difference is caused by the larger beamwidth and $\alpha$ of AMSU-A, for the Moon remains longer in a larger field of view (FOV), and the circumference of the circle described in the sky by the DSV direction is smaller for a larger $\alpha$. The exact moments in time when the Moon enters the DSV have to be calculated with ephemeris. Depending on the orbital elements, the small number of Moon intrusions varies among different years.

\section{Uncertainties of the reference flux density from the Moon}

Even though the Moon appears several times per year in the DSV of microwave sensors, and models are available for subtracting the contribution of this natural satellite to the reference flux from the CMB, this object has not been used as a calibration standard at millimeter wavelengths yet. The main reasons are imperfections of the calculations of the tempera- 


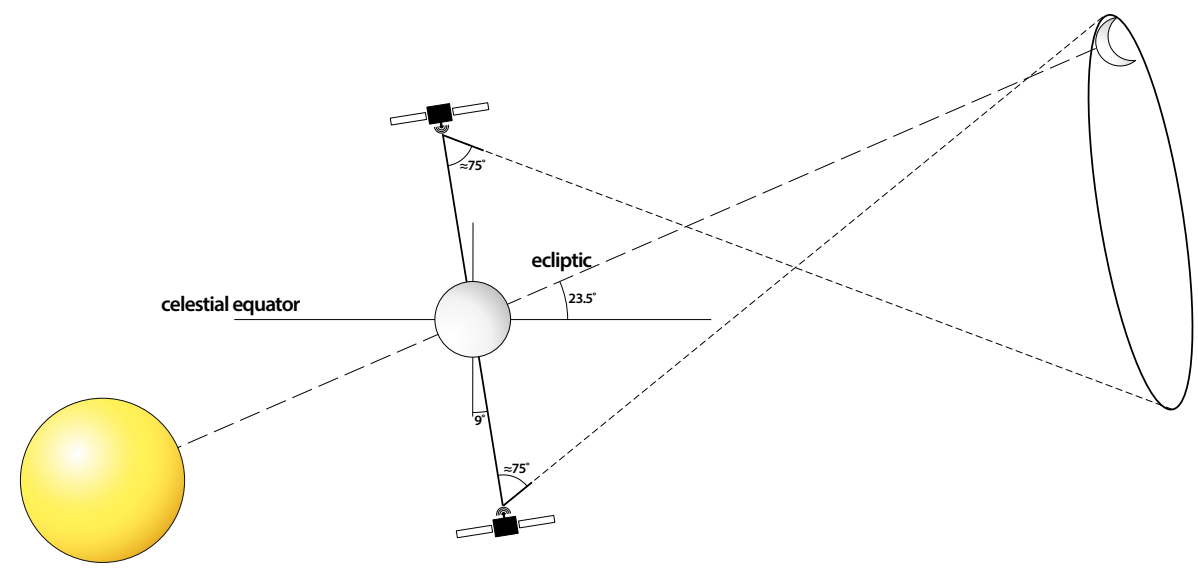

Figure 1. Viewing direction of the deep-space view (DSV, short dashed line) compared to the celestial equator and the ecliptic plane (long dashed line). For simplicity, the slight tilt of the Moon's orbit against the ecliptic is not displayed. The DSV direction has a typical angle $\alpha \approx 75^{\circ}$ against nadir and describes a circle in the sky during one orbit.

Table 1. Frequency of appearances of the Moon in the deep-space view for different instruments. The third column gives the angle(s) between the orbital axis of the artificial satellite and the DSV position(s). Intrusions affect several orbits in a row (see Table 2); the fifth column gives their frequency.

\begin{tabular}{llrrrr}
\hline Satellite & Instrument & $90^{\circ}-\alpha$ & Period considered & Intrusion frequency & Time of ascending node (LT) \\
\hline MetOp-A & MHS & $14.2-18.6^{\circ}$ & Dec 2006-Dec 2015 & 7.8 year $^{-1}$ & $21: 30$ \\
NOAA-15 & AMSU-B & $9-13^{\circ}$ & Jan 2006-Dec 2010 & 4 year $^{-1}$ & $16: 30-18: 00$ \\
Suomi NPP & ATMS & $4.4-8.8^{\circ}$ & Jan 2013-Apr 2016 & 4.5 year $^{-1}$ & $13: 30$ \\
NOAA-15 & AMSU-A & $6.7^{\circ}$ & May 2014-Apr 2016 & 4 year $^{-1}$ & $17: 00-18: 00$ \\
NOAA-15 & AMSU-A & $6.7^{\circ}$ & Jan 2006-Dec 2010 & 2.2 year $^{-1}$ & $16: 30-18: 00$ \\
\hline
\end{tabular}

ture of the Moon and systematic errors caused by the fact that the Moon fills only a fraction of the FOV of the instrument.

\subsection{Radiant flux of the Moon}

The algorithm used by Mo and Kigawa (2007) for detection and correction of the lunar contamination in AMSUA, which was later modified by Yang and Weng (2016) for ATMS (Advanced Technology Microwave Sounder), allows calculating the brightness of the Moon under the assumptions that the disk-integrated emissivity of the surface is 0.95 , independent of the apparent "geodetic" longitude and latitude of the center of the Moon seen by the sensor. The diskintegrated temperature of the Moon is here a quadratic function of $\cos \Theta, \Theta$ being the Sun-satellite-Moon angle. This picture could not make allowance for the complex structure of the lunar surface that was recently revealed at frequencies similar to those of channels 1 and 2 of AMSU-A by China's first lunar probe, Chang'e-1 (Zheng et al., 2012). A more sophisticated model of the microwave radiation from the Moon, however, would require well-calibrated observations of the Moon for years, in regular intervals and at a variety of frequencies, just like they have been carried out at visible wavelengths. As the Earth's atmosphere is for the most part transparent to microwaves, such an observing program is in principle feasible but has to our best knowledge not been undertaken yet.

The range of possible angles $\Theta$ can be estimated according to the following formula. It is not quite accurate, because the orbit of the Moon is tilted against the ecliptic. Obviously $\Theta$ cannot be larger than $180^{\circ}$.

$\Theta_{\min } \approx \mathrm{ECT}+\alpha$

$\mathrm{ECT} \leq \alpha \Rightarrow \Theta_{\max } \approx \mathrm{ECT}+180^{\circ}-\alpha$

$\mathrm{ECT} \geq \alpha \Rightarrow \Theta_{\max } \approx 180^{\circ}$

ECT is the absolute value of the solar hour angle when and where the spacecraft crosses the Equator in the daytime.

MetOp-A, for example, crosses the Equator at 09:30 LT (local time); i.e., its ECT $=2.5 \times 15^{\circ}=37.5^{\circ}$. With an $\alpha$ of $71.4^{\circ}$ for MHS, this means that the approximate range of $\Theta$ is 109 to $146^{\circ}$. As the Equator-crossing time drifts during the lifetime of most other satellites, the phase angles of the Moon during an intrusion in the DSV vary as well between the start and end of the mission. 
Table 2. Date and time of the closest approaches of the Moon to the deep-space view of two microwave sounders in the year 2007. The third and seventh column give the position of the DSV direction relative to the celestial equator at these moments in time, and the fourth and eighth column give the number of orbits which were contaminated by the Moon. Intrusion events affect several orbits in a row, sometimes in groups of two with 1 or 2 days in between. Their durations depend on the relative movements of the DSV direction and the Moon. Due to a few simplifications in the calculations, the values in this table are only accurate to $1^{\circ}$ and $1 \mathrm{~h}$.

\begin{tabular}{|c|c|c|c|c|c|c|c|}
\hline \multicolumn{4}{|c|}{ AMSU-B on NOAA-15 } & \multicolumn{4}{|c|}{ MHS on MetOp-A } \\
\hline Date & Time & Declination & Orbits affected & Date & Time & Declination & Orbits affected \\
\hline 3 Jan & 00:00 & $28^{\circ}$ & 0 & 6 Jan & $14: 00$ & $17^{\circ}$ & 9 \\
\hline $1 \mathrm{Feb}$ & $14: 00$ & $23^{\circ}$ & 0 & $6 \mathrm{Feb}$ & 07:00 & $-2^{\circ}$ & 34 \\
\hline 3 Mar & 18:00 & $8^{\circ}$ & 8 & $8 \mathrm{Mar}$ & 02:00 & $-16^{\circ}$ & 0 \\
\hline $2 \mathrm{Apr}$ & 01:00 & $-3^{\circ}$ & 45 & $6 \mathrm{Apr}$ & $21: 00$ & $-25^{\circ}$ & 0 \\
\hline 1 May & $15: 00$ & $-15^{\circ}$ & 0 & 6 May & $16: 00$ & $-28^{\circ}$ & 0 \\
\hline 31 May & 04:00 & $-25^{\circ}$ & 0 & 5 Jun & $11: 00$ & $-21^{\circ}$ & 0 \\
\hline 29 Jun & $18: 00$ & $-28^{\circ}$ & 0 & $5 \mathrm{Jul}$ & 07:00 & $-7^{\circ}$ & 15 \\
\hline $29 \mathrm{Jul}$ & 08:00 & $-24^{\circ}$ & 0 & 3 Aug & 07:00 & $5^{\circ}$ & $8+7$ \\
\hline 27 Aug & $22: 00$ & $-13^{\circ}$ & 0 & 31 Aug & 09:00 & $10^{\circ}$ & 8 \\
\hline $26 \mathrm{Sep}$ & $12: 00$ & $1^{\circ}$ & 18 & 30 Sep & 02:00 & $23^{\circ}$ & 16 \\
\hline 25 Oct & $15: 00$ & $13^{\circ}$ & $7+7$ & 29 Oct & $15: 00$ & $28^{\circ}$ & 0 \\
\hline $23 \mathrm{Nov}$ & 18:00 & $23^{\circ}$ & 0 & $28 \mathrm{Nov}$ & 04:00 & $24^{\circ}$ & 28 \\
\hline $23 \mathrm{Dec}$ & 04:00 & $28^{\circ}$ & 0 & $27 \mathrm{Dec}$ & $13: 00$ & $15^{\circ}$ & $8+8$ \\
\hline
\end{tabular}

\subsection{Uncertainties of the antenna pattern and the Moon's position in the beam}

Because the nominal $3 \mathrm{~dB}$ beamwidth is $3.3^{\circ}$ for AMSU-A and $1.1^{\circ}$ for AMSU-B and MHS, the full disk of the Moon with an equatorial angular width between $29.4^{\prime}$ and $33.5^{\prime}$ fills only part of the DSV. It is therefore very important to know exactly where the Moon is relative to the center of the beam. This angle is supposed to be known with an accuracy of $0.3^{\circ}$ according to the MHS Level 1 Product Generation Specification. Right ascension and declination of the Moon as seen from Earth are of course known with much higher accuracy, but these coordinates have to be transformed to the position of the spacecraft and the instrument FOV reference frame. In addition to the error in the Moon calculation one has to consider the line-of-sight offset of the DSV. With AMSU$\mathrm{B}$, for example, the pointing of the antenna is known within $0.05^{\circ}$, contributing only a negligible uncertainty compared to the assumed error in the position of the Moon (Charlton and Jarrett, 1993). Assuming that the antenna beam patterns are approximately Gaussian, a distance from the center of $0.3^{\circ}$ translates to a loss of antenna efficiency of $2 \%$ for AMSU-A and $20 \%$ for AMSU-B and MHS with their smaller beams. As the Moon is an extended source, these values are lower limits for the actual loss in signal. The $3 \mathrm{~dB}$ beamwidth itself is only known with an accuracy of $10 \%$ for AMSU-B (Atkinson, 2001). This means an additional loss in signal of $14 \%$ if a point source is actually $10 \%$ further away from the center of the beam than the assumed $3 \mathrm{~dB}$ beamwidth.

Figure 2 shows a typical light curve of the Moon in different DSVs. Its minimum distance from the center of the FOV is different for each DSV, because they point in slightly different directions. The maximum signal is reached at closest approach, so the time difference between the minima in the upper panel of Fig. 2 and the maxima in the lower panel is indicative of the actual pointing error of the satellite. It amounts to some $10 \mathrm{~s}$ for DSV 2 in Fig. 2; this is among the larger values we could find from examining a few dozen intrusions of the Moon. From the angular velocity of the DSV direction in the sky, $\omega$, we obtain the typical pointing error of MHS.

$\omega=2 \pi \cdot\left(90^{\circ}-\alpha\right) / 6000 \mathrm{~s} \approx 0.017^{\circ} \mathrm{s}^{-1}$

This means that the error in the Moon calculation is in most cases smaller than $0.2^{\circ}$, i.e., smaller than assumed in the MHS Level 1 Product Generation Specification. The movement of the Moon itself with an angular velocity of $1.4^{\circ} \times 10^{-4} \mathrm{~s}^{-1}$ was neglected.

\section{Benefits from measuring the Moon signal during a pitch-over maneuver}

\subsection{Reduction of errors and uncertainties}

The uncertainties described in Sect. 3.2 can be greatly reduced if dedicated observations of the Moon are used to check the stability of the calibration of microwave sensors, because they are carried out at the same phase angle and with the Moon in the center of the beam. The exact position of the Moon can be determined with a small raster map if necessary.

Flux variations due to changes in distance between the sensor and the Moon can be corrected for with an inverse square law; those due to changes in distance between the Sun and 

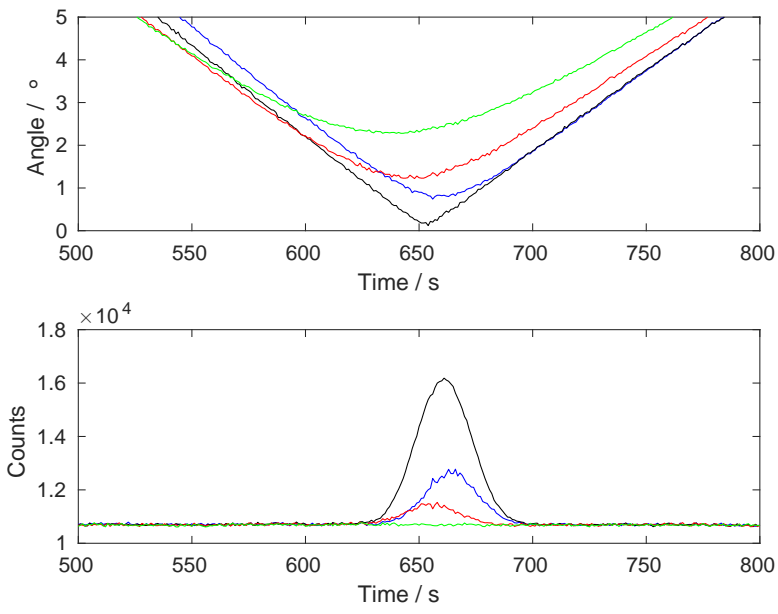

Figure 2. Moon intrusion event in different deep-space views on 30 October 2015, with MHS on MetOp-A; blue: DSV 1; black: DSV 2; red: DSV 3; green: DSV 4. Top: angle between Moon and space view. Bottom: space view count.

the Moon, however, are more difficult to handle. They can be estimated with the Stefan-Boltzmann law:

$d_{\mathrm{SM}}^{-2} \propto F_{\text {in }}=F_{\text {out }} \propto T_{\text {eff }}^{4}$,

where $F_{\text {in }}$ is the flux absorbed by the Moon, $F_{\text {out }}$ is the flux emitted by the Moon, $d_{\mathrm{SM}}$ is the distance between the Sun and the Moon, and $T_{\text {eff }}$ is the effective temperature of the Moon.

Flux changes due to variations of longitude and latitude of the center of the lunar disk are difficult to disentangle from the larger effects caused by the variations of phase angle. The period of libration corresponds rather to the anomalistic than the synodic month, and its maximum value is $7^{\circ} 53^{\prime}$ in longitude and $6^{\circ} 40^{\prime}$ in latitude. Because the strongest longitudinal variations in microwave emission are found near $\lambda=0^{\circ}$ (Zheng et al., 2012), however, these areas are always present on the lunar disk, independent of the exact "sub-observer" point. The situation is similar in the thermal infrared, where libration in latitude causes a systematic error of less than $1 \%$ (Daniels et al., 2015). Unlike random appearances in the DSV, pointing the instrument at the Moon only at a certain phase with dedicated maneuvers of the satellite therefore eliminates the dominating component of the Moon's flux variations.

\subsection{Lifetime stability}

The main aim of observations of the Moon is a check of the stability of the photometric calibration. Its standard procedure converts Earth view counts to radiance by the aid of repeated viewings of the onboard blackbody target and cold space. The assumed flux from the former, however, can be affected by time-dependent systematic errors, e.g., inaccurate thermometers or changing emissivity. It is therefore desirable to establish a "reference for the reference" that is not affected by wear and tear. Given the fact that the gain of a microwave sounder can change by a factor of 10 during the mission, as happened with AMSU-B on NOAA-16, it is not enough to rely on the cosmic microwave background as the only reference immune to deterioration. Such a "higherorder" standard should be observed at least at the start and the end of the mission in order to detect monotonous drifts. The higher the number of such spacecraft maneuvers, the better the characterization of the stability of the sensor. Therefore lunar observations were carried out some 10 times per month at or near opposition with the Clouds and the Earth's Radiant Energy System (CERES) in order to provide enough data for reducing the remaining uncertainties, e.g., those caused by libration. Daniels et al. (2015) were then able to detect trends of $1 \%$ per decade or less in the infrared channels of CERES.

\subsection{Examples: Ice Cloud Imager and Microwave Imager on EPS-SG}

There will be two microwave imaging radiometers for MetOp-SG: ICI and MWI. Unlike AMSU-B and MHS they are imagers, and they will carry out conical instead of crosstrack scans (Alberti et al., 2012). Another major difference concerns the frequency channels, which are compared to those of other microwave sensors in Table 3. The use of conical scans makes lunar observations with pitch-over maneuvers even more useful, because they will allow both deep space and the Moon to be observed without the cold calibration reflector, whose optical properties introduce an additional error source in the routine calibration of every scan. With a small raster map, it will also be possible to measure the antenna pattern in flight and to monitor its stability. The accuracy requirement of these instruments is $0.5 \mathrm{~K}$ (Alberti et al., 2012), and the random uncertainty can be reduced by combining on the order of 100 scans at each point of the raster, for each scan only lasts a few seconds. The instantaneous FOV is smaller than that of MHS $-0.65^{\circ}$ vs. $1.11^{\circ}$ - so one can expect a $(1.11 / 0.65)^{2} \approx 2.9$ times higher signal from the Moon compared to microwave background and blackbody. A FOV of $0.65^{\circ}$ is still sufficient to cover the full disk of the Moon even at its smallest distance from Earth, but it requires a pointing accuracy of $\left(0.65-0.5^{\circ}\right) / 2=0.075^{\circ}$ to make sure the Moon is within the borders of the $3 \mathrm{db}$ beamwidth. Hence the step size of the abovementioned raster map should not be larger than a few arc minutes. We advocate using the Moon for checking the photometric stability of all channels of ICI and MWI except for the lowest frequencies of MWI, where solar coronal activity might significantly increase the flux from the Sun reflected by the Moon to the effect that it can no longer be neglected. 
Table 3. Channel frequencies in GHz of different microwave instruments. The values were obtained from Goodrum et al. (2014), Weng et al. (2013), and Alberti et al. (2012).

\begin{tabular}{|c|c|c|c|c|}
\hline AMSU & MHS & ATMS & ICI & MCI \\
\hline & & & & 18.7 \\
\hline 23.8 & & 23.8 & & 23.8 \\
\hline 31.4 & & 31.4 & & 31.4 \\
\hline 50.3 & & 50.3 & & 50.3 \\
\hline \multirow[t]{2}{*}{52.8} & & 51.76 & & 52.6 \\
\hline & & 52.8 & & 53.2 \\
\hline $53.596 \pm 0.115$ & & $53.596 \pm 0.115$ & & 53.8 \\
\hline 54.4 & & 54.4 & & \\
\hline 54.94 & & 54.94 & & \\
\hline 55.5 & & 55.5 & & \\
\hline 57.29 & & 57.290344 & & \\
\hline $57.29 \pm 0.217$ & & $57.290344 \pm 0.217$ & & \\
\hline $57.29 \pm 0.3222 \pm 0.048$ & & $57.290344 \pm 0.3222 \pm 0.048$ & & \\
\hline $57.29 \pm 0.3222 \pm 0.022$ & & $57.290344 \pm 0.3222 \pm 0.022$ & & \\
\hline $57.29 \pm 0.3222 \pm 0.01$ & & $57.290344 \pm 0.3222 \pm 0.01$ & & \\
\hline $57.29 \pm 0.3222 \pm 0.0045$ & & $57.290344 \pm 0.3222 \pm 0.0045$ & & \\
\hline 89 & 89 & $87-91$ & & 89 \\
\hline \multirow[t]{4}{*}{$89.9 \pm 0.9$} & & & & 100.49 \\
\hline & & & & $118.7503 \pm 2.1$ \\
\hline & & & & $118.7503 \pm 1.4$ \\
\hline & & & & $118.7503 \pm 1.2$ \\
\hline $150 \pm 0.9$ & 157 & 166.31 & & 166.9 \\
\hline \multirow[t]{2}{*}{$183.31 \pm 1$} & $183.311 \pm 1$ & $183.31 \pm 1$ & & $183.31 \pm 2$ \\
\hline & & $183.31 \pm 1.8$ & $183.31 \pm 2$ & $183.31 \pm 3.4$ \\
\hline \multirow[t]{2}{*}{$183.31 \pm 3$} & $183.311 \pm 3$ & $183.31 \pm 3$ & & $183.31 \pm 4.9$ \\
\hline & & $183.31 \pm 4.5$ & $183.31 \pm 4.9$ & $183.31 \pm 6.1$ \\
\hline \multirow[t]{9}{*}{$183.31 \pm 7$} & 190.311 & $183.31 \pm 7$ & $183.31 \pm 8.4$ & $183.31 \pm 8.4$ \\
\hline & & & $243.2 \pm 2.5$ & \\
\hline & & & $325.15 \pm 9.5$ & \\
\hline & & & $325.15 \pm 3.5$ & \\
\hline & & & $325.15 \pm 1.5$ & \\
\hline & & & $448 \pm 7.2$ & \\
\hline & & & $448 \pm 3$ & \\
\hline & & & $448 \pm 1.4$ & \\
\hline & & & $664 \pm 4.2$ & \\
\hline
\end{tabular}

Table 4. Moon intrusion events observed with MHS where the image of the Moon crossed the center of the field of view. The size of the various corrections applied in calculating the maximum signal in each light curve is given. This signal is normalized so that the value measured with NOAA-19 is 1.

\begin{tabular}{llrrrrr}
\hline Satellite & Date & Time (UT) & Counts/gain & Eq. diam. of Moon & $T_{\mathrm{B}}^{\text {Moon }}$ & Maximum signal \\
\hline NOAA-19 & 22 Dec 2015 & $13: 12$ & $32.3 \mathrm{~K}$ & $1946^{\prime \prime}$ & $295 \mathrm{~K}$ & 1 \\
NOAA-18 & 22 Jan 2016 & $12: 33$ & $31.8 \mathrm{~K}$ & $1875^{\prime \prime}$ & $319 \mathrm{~K}$ & 0.979 \\
NOAA-18 & 1 Apr 2015 & $05: 20$ & $26.4 \mathrm{~K}$ & $1765^{\prime \prime}$ & $300 \mathrm{~K}$ & 0.979 \\
NOAA-18 & 19 Nov 2007 & $08: 22$ & $25.8 \mathrm{~K}$ & $1951^{\prime \prime}$ & $234 \mathrm{~K}$ & 1.003 \\
NOAA-18 & 8 Apr 2006 & $16: 56$ & $24.7 \mathrm{~K}$ & $1769^{\prime \prime}$ & $268 \mathrm{~K}$ & 1.017 \\
NOAA-18 & 9 Jan 2006 & $18: 31$ & $27.4 \mathrm{~K}$ & $1851^{\prime \prime}$ & $269 \mathrm{~K}$ & 1.023 \\
\hline
\end{tabular}



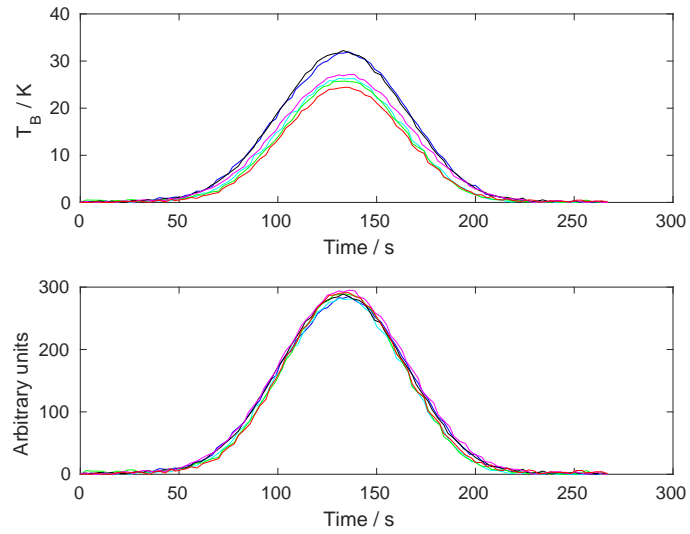

Figure 3. Moon intrusion events observed with channel H1 of MHS (magenta: NOAA18, 9 January 2006; red: NOAA18, 8 April 2006; green: NOAA18, 19 November 2007; cyan: NOAA18, 1 April 2015; blue: NOAA18, 22 January 2016; black: NOAA19, 22 December 2015). Top: counts divided by gain. Bottom: counts divided by gain and scaled with the inverse of the solid angle and brightness temperature of the Moon. The counts corresponding to the CMB level were subtracted.

\subsection{Lifetime stability of ongoing missions}

In the absence of dedicated pitch-over maneuvers one has to compare unplanned intrusion events where the Moon crossed the center of the FOV. This condition is fulfilled for those positions of the DSV whose neighbors have identical light curves. Figure 3 shows the light curves from such comparable intrusion events, and Table 4 gives the maximum signal before and after correction for systematic effects. We find an upper limit for the lifetime stability of $2 \%$ with just two corrections: Earth-Moon distance and phase angle (according to Mo and Kigawa, 2007). As changes in the Moon's radiance can be characterized with much higher accuracy than its absolute radiance, we have arbitrarily chosen the value measured with NOAA-19 as a reference. The good agreement between the instruments on NOAA-18 and NOAA-19 means in particular that their beam characteristics must be almost identical; this is not the case for all MHSs. Because of the small eccentricity of the orbit of the Earth (0.017), no correction for the distance to the Sun was applied.

Contrary to expectations (Mo and Kigawa, 2007), the Moon can contaminate all four DSVs of MHS at the same time (see Fig. 4), though the two outer positions are only very slightly affected. This kind of event can happen in several orbits per year and is best dealt with by interpolating between the counts before and after the anomaly.

\section{Other intruders in the deep-space view}

The Moon is the brightest solar system object except for the Sun. Mo and Kigawa (2007) estimate its brightness temper-

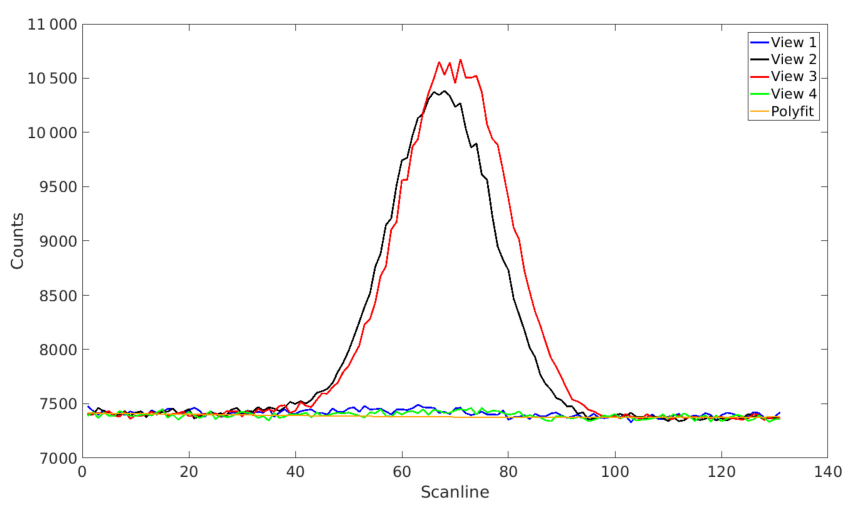

Figure 4. Moon intrusion event in different deep-space views on 17 July 2015 with MHS. The position of the Moon falls almost exactly between DSV 2 and 3 just so it affects the counts in all four positions. The orange line gives the number of counts in views 1 and 4 without any contribution from the Moon; it falls below the actually measured count levels from all four space views during the closest approach of the Moon.

ature to be $258 \mathrm{~K}$ at all frequencies for $\Theta=127.5^{\circ}$, a typical value for Moon intrusions with MHS on MetOp-A and MetOp-B. This means that the increase of counts from the DSV caused by the intrusion of the Moon is a bit less than the fraction of the FOV covered by the Moon multiplied by the count difference between DSV and black body level (see Table 5).

Table 5 also contains entries for two other sources: Jupiter, the brightest radio source among the outer planets, and the Crab Nebula, a supernova remnant that strongly emits synchrotron radiation. It can be seen that for the currently operational microwave satellites neither object poses a problem in terms of contamination of the DSV: at $23.8 \mathrm{GHz}$ and a FOV with $3.3^{\circ}$ diameter, the contribution is smaller than the resolution of the analog-to-digital converter; at $183.3 \mathrm{GHz}$ and a FOV with $1.1^{\circ}$ diameter, the contribution is about an order of magnitude smaller than the noise. This might change with ICI and MWI, however, because their FOVs are smaller than for previous sensors, and therefore the brightness of any source entering the DSV relative to the black body signal increases.

\section{Conclusions}

With decreasing beamwidth at $183 \mathrm{GHz}-3.3^{\circ}$ for SSM/T$2,1.1^{\circ}$ for AMSU-B, and $0.65^{\circ}$ for MWI - the signal of the Moon at its appearances in the deep-space view has increased relative to the contribution from the cosmic microwave background. As a consequence, it will be possible for future missions to measure the lunar flux with sufficient accuracy to check whether the requirements on the stability of the instrument are fulfilled. While the emissivity of the Moon itself can confidently be assumed to be constant, it is essential to 
Table 5. Brightness of different astronomical sources. No brightness temperatures are given for the Crab Nebula, for this is not thermal emission. The flux densities $\Phi$ in the fifth and sixth column are normalised to the black body. The values were calculated for two channels with very different frequencies on AMSU-A and MHS; the exposures of DSV and black body differ typically by 3500 counts for channel 1 of AMSU-A and 47000 counts for channel 3 of MHS.

\begin{tabular}{lrrrrrl}
\hline Object & $T_{\mathrm{B}}^{23.8 \mathrm{GHz}}$ & $T_{\mathrm{B}}^{183.3 \mathrm{GHz}}$ & Diameter & $\Phi$ at $24 \mathrm{GHz}$ & $\Phi$ at $183 \mathrm{GHz}$ & Ref. \\
\hline $\mathrm{BB}$ & $280 \mathrm{~K}$ & $280 \mathrm{~K}$ & - & 1 & 1 & \\
$\mathrm{CMB}$ & $2.72548 \mathrm{~K}$ & $2.72548 \mathrm{~K}$ & - & $7.9 \times 10^{-3}$ & $1.3 \times 10^{-3}$ & Fixsen (2009) \\
Moon & $258 \mathrm{~K}$ & $258 \mathrm{~K}$ & $30 \operatorname{arcmin}$ & $2.1 \times 10^{-2}$ & $1.9 \times 10^{-1}$ & Mo and Kigawa (2007) \\
Jupiter & $130 \mathrm{~K}$ & $150 \mathrm{~K}$ & 0.8 arcmin & $7.6 \times 10^{-6}$ & $7.8 \times 10^{-5}$ & Spohn et al. (2014) \\
Crab Nebula & $S=400 \mathrm{Jy}$ & $\mathrm{S}=290 \mathrm{Jy}$ & $10 \operatorname{arcmin}$ & $3.2 \times 10^{-5}$ & $2.3 \times 10^{-6}$ & Macias-Perez et al. (2010), \\
& & & & & & Mezger et al. (1986) \\
\hline
\end{tabular}

minimize the uncertainties related to the dependence of the flux on phase angle and the position of the Moon in the field of view. Both sources of error are best addressed with dedicated spacecraft maneuvers. If such maneuvers also include a raster map, one can characterize the beam pattern in flight. For this purpose, however, deconvolution of the images will become necessary, because the Moon is an extended source, especially for ICI and MWI. A raster map would also aid in determining the exact position of the Moon. If an accuracy of $1 / 10$ of the $3 \mathrm{db}$ bandwidth could be achieved, the corresponding flux error would amount to only a few percent.

Its invariability makes the Moon also well suited for intercalibration between sensors that were operational at quite different epochs. This requires identical beam patterns of the sensors, an assumption that can be checked by means of the light curves themselves. Given the fact that the unplanned lunar intrusions in the deep-space view happen over a wide range of phase angles, because of orbital drifts and different viewing geometries, it is quite possible to find pairs of observations with similar phase angles from different satellites. This way one eliminates the unavoidable differences in scene temperature that adversely affect other methods like simultaneous nadir overpasses and zonal averages. A ground-based observing program of the Moon at the wavelengths of the window channels would considerably reduce the errors associated with remaining phase angle differences and libration. Microwave sensors in space, however, also operate at wavelengths for which the Earth's atmosphere is opaque. Furthermore, Zheng et al. (2012) showed that the brightness temperature of the warmest features on the lunar surface can vary by $30 \mathrm{~K}$ between different frequencies. Hence a complete model of the brightness of the Moon should allow for variations with frequency. Observations of the Moon carried out from an airplane flying in the stratosphere, e.g., ISMAR (International SubMillimeter Airborne Radiometer; Fox et al., 2014), would avoid $99 \%$ of the water vapor in the Earth's atmosphere. Unfortunately, however, the Moon can only cover a small fraction of the beam of ISMAR, which has a halfpower width of some $5^{\circ}$. Therefore ground-based millimeter observatories are a more promising alternative. They can eas- ily resolve the lunar disk spatially, but obviously they cannot be used for observations of the Moon at $183.3 \mathrm{GHz}$.

While the Moon produces thousands of counts when moving through the deep-space view, the possible impact from other discreet sources in the sky on the low-level reference flux from the cosmic microwave background is orders of magnitude smaller. Even with next-generation instruments, intrusions in the DSV from objects other than the Moon will have no significant impact on the calibration accuracy in the microwave range.

\section{Data availability}

The data from AMSU and MHS are available from NOAA CLASS, http://www.class.ngdc.noaa.gov/saa/products/ welcome. Some of them can be displayed with STAR ICVS, http://www.star.nesdis.noaa.gov/icvs/InstrPerfMonitoring. php. Phase angles of the Moon were calculated with JPL's HORIZONS system, http://ssd.jpl.nasa.gov/horizons.cgi.

Author contributions. S. Buehler started and guided this investigation. I. Hans, T. Lang, and S. Michel developed the MATLAB code for reading the MHS Level $1 \mathrm{~b}$ science packets. T. Lang and $\mathrm{S}$. Michel identified instances of intrusions of the Moon in the deepspace views of the microwave sensors. M. Burgdorf prepared the manuscript with contributions from all co-authors.

Acknowledgements. This work was undertaken within the project "Fidelity and Uncertainty in Climate data records from Earth Observation (FIDUCEO)". FIDUCEO has received funding from the European Union's H2020 Research and Innovation program, under Grant Agreement 638822. The authors wish to thank Nigel Atkinson and Oliver Lemke for helpful tips on using the ATOVS and AVHRR Pre-processing Package.

Edited by: S. J. Munchak

Reviewed by: two anonymous referees 


\section{References}

Alberti, G., Memoli, A., Pica, G., Santovito, M. R., Buralli, B., Varchetta, S., d'Addio, S., and Kangas, V.: TWO Microwave Imaging radiometers for MetOp Second Generation, Proc. of the 2012 CNIT Tyrrhenian Workshop, 12-14 September 2012.

Atkinson, N. C.: Calibration, Monitoring and Validation of AMSUB, Adv. Space Res., 28, 117-126, 2001.

Charlton, J. E. and Jarrett, M. L.: Antenna Pattern Measurements on AMSU-B, P. Soc. Photo-Opt. Ins., 1935, 199-208, 1993.

Charlton, J. E., Jarrett, M. L., and Tatnall, A. R. L.: Assessment of calibration errors on AMSU-B, P. Soc. Photo-Opt. Ins., 1935, 160-168, 1993.

Coates, R. J.: Lunar Brightness Variations with Phase at 4.3-MM Wave Length, Astrophys. J, 133, 723-725, 1961.

Daniels, J. L., Smith, G. L., Priestley, K. J., Thomas, S.: Using Lunar Observations to Validate In-Flight Calibrations of Clouds and the Earth's Radiant Energy System Instruments, IEEE T. Geosci. Remote S., 53, 5110-5116, 2015.

Fixsen, D. J.: The Temperature of the Cosmic Microwave Background, Astrophys. J., 707, 916-920, 2009.

Fox, S., Lee, C., Rule, I., King, R., Rogers, S., Harlow, C., and Baran, A.: ISMAR, Proc. 13th Specialist Meeting on Microwave Radiometry and Remote Sensing of the Environment, 128-132, 24-27 March 2014.

Goodrum, G., Kidwell, K. B., and Winston, W.: NOAA KLM user's guide - August 2014 Revision, Tech. rep., National Environmental Satellite, Data, and Information Services, 2014.

John, V. O., Holl, G., Buehler, S. A., Candy, B., Saunders, R. W., and Parker, D. E.: Understanding intersatellite biases of microwave humidity sounders using global simultaneous nadir overpasses, J. Geophys. Res.-Atmos., 117, D02305, doi:10.1029/2011JD016349, 2012.

John, V. O., Allan, R. P., Bell, W., Buehler, S. A., and Kottayil, A.: Assessment of intercalibration methods for satellite microwave humidity sounders, J. Geophys. Res.-Atmos., 118, 4906-4918, 2013.

JPL's HORIZONS system: JPL Solar System Dynamics, available at: http://ssd.jpl.nasa.gov/horizons.cgi, last access: 27 May 2016.

Karl, Th. R., Arguez, A., Huang, B., Lawrimore, J. H., McMahon, J. R., Menne, M. J., Peterson, Th. C., Vose, R. S., and Zhang, H.M.: Possible artefacts of data biases in the recent global surface warming hiatus, Science, 348, 1469-1472, 2015.
Kieffer, H. H.: Photometric Stability of the Lunar Surface, Icarus, 130, 323-327, 1997.

Kieffer, H. H. and Stone, Th. C.: The Spectral Irradiance of the Moon, Astron. J., 129, 2887-2901, 2005.

Kieffer, H. H. and Wildey, R. L.: Establishing the Moon as a Spectral Radiance Standard, J. Atmos. Ocean. Tech., 13, 360-375, 1996.

Macias-Perez, J. F., Mayet, F., Aumont, J., and Desert, F.-X.: Global Spectral Energy Distribution of the Crab Nebula in the Prospect of the Planck Satellite Polarisation Calibration, Astrophys. J., 711, 417-424, 2010.

Mezger, P. G., Tuffs, R. J., Chini, R., Kreysa, E., and Gemünd, H.-P.: Maps of Cassiopeia A and the Crab nebula at $\lambda 1.2 \mathrm{~mm}$, Astron. Astrophys., 167, 145-150, 1986.

Mo, T. and Kigawa, S.: A study of lunar contamination and on-orbit performance of the NOAA 18 Advanced Microwave Sounding Unit A, J. Geophys. Res.-Atmos., 112, D20124, doi:10.1029/2007JD008765, 2007.

NOAA CLASS: NOAA Level 1b data sets, available at: http://www. class.ngdc.noaa.gov/saa/products/welcome, NOAA/NESDIS Suitland, Maryland, USA, last access: 5 February 2016.

Patt, F. S., Eplee, R. E., Barnes, R. A., Meister, G., and Butler, J. J.: Use of the Moon as a calibration reference for NPP VIIRS, P. Soc. Photo-Opt. Ins., 588215, 1-12, 2005.

Spohn, T., Breuer, D., and Johnson, T. V.: Encyclopedia of the Solar System, Elsevier, Amsterdam, the Netherlands, 2014, p. 1113 , ISBN-13: 978-0-12-415845-0, 2014.

STAR ICVS: NOAA STAR, available at: http://www.star. nesdis.noaa.gov/icvs/InstrPerfMonitoring.php, last access: 27 May 2016.

Weng, F., Zou, X., Sun, N., Yang, H., Tian, M., Blackwell, W. J., Wang, X., Lin, L., and Anderson, K.: Calibration of Suomi national polar-orbiting parterneship advanced technology microwave sounder, J. Geophys. Res.-Atmos., 118, 1-14, 2013.

Yang, H. and Weng, F.: Corrections for On-Orbit ATMS Lunar Contamination, IEEE T. Geosci. Remote S., 54, 1918-1924, 2016.

Zheng, Y. C., Tsang, K. T., Chan, K. L., Zou, Y. L., Zhang, F., and Ouyang, Z. Y.: First microwave map of the Moon with Chang'E1 data, Icarus, 219, 194-210, 2012. 